\title{
Analysis and Design of Employment and Recruitment System based on
}

\author{
UML \\ Hong-jie TANG \\ Department of Vocational Education, Liaoning Police Academy, Dalian, China \\ thongjie@163.com
}

Key Words: employment; recruitment; UML

\begin{abstract}
Employment and recruitment system based on UML provides a virtual and intelligent talent market for employers and job hunters, which can shorten their distance quickly. According to the different permissions, the users may take different operations to meet their respective demands. This paper develops an employment and recruitment system with UML by object oriented analysis and design methods, which includes requirement analysis, class design and interactive design. In the development progress, system use-case diagram, management class diagram, job search class diagram, recruitment class diagram, register sequence diagram and modification activity diagram are displayed in detail.
\end{abstract}

\section{Introduction}

In the age of rapid development, companies have an increasing need of talents. And the "employment and recruitment system" emerged in this context1. Users can sign up in the system as a job hunter, and employer or manager2 according to their own needs or permissions use the functions provided by the system.

It is found through the survey and analysis that there are such basic modules in the "employment and recruitment system": Job Searching, Recruitment and Management3. The functions of these modules are listed as follows:

1) In Job Searching, users can update job-hunter's profile, search for employment info, release their job intension, hand in resumes and view their application e-mail.

2) In Recruitment, users can update employer's profile, search for application info, release employment info, browse applicant's resumes and reply applicants.

3) In Management, users can update manager's profile, manage job hunters, employers and news.

\section{Requirement Analysis}

1) Analysis of system participants

According to the methods of identifying participants, main participants preliminarily found in the "employment and recruitment system" include User, Seeker (job hunter), Inviter (employer), and Administrator.

2) Analysis of use cases

On the basis of main participants (User, Seeker, Inviter, Administrator) found in the system, chief use cases of "employment and recruitment system" preliminarily identified include: Register, Login, Modify Info, Seek Job, Issue Application, Post Resume, Browse SeekMail, Search SeekInfo, Issue Invitation, Browse Resume, Browse InviteMail, Reply Seeker, Manage Seeker, Manage 
Inviter and Manage News.

3) Analysis of the relationship in use case models

Obviously, there's association relationship between the participants (including User, Seeker, Inviter, and Administrator) and their own use cases. And there is including relationship between the use case of Login and the use case of Register. Similarly, there is including relationship between use case of "Modify Info" and the use case of "Login" too. And it's sure that there's generalization relationship among Seeker, Inviter, Administrator and Users.

Use case diagram is the main UML diagram4 adopted in the process of needs analysis. Based on the analysis as above, the general use case diagram of the "employment and recruitment system" is drawn with Rational Rose as shown in Fig. 1.

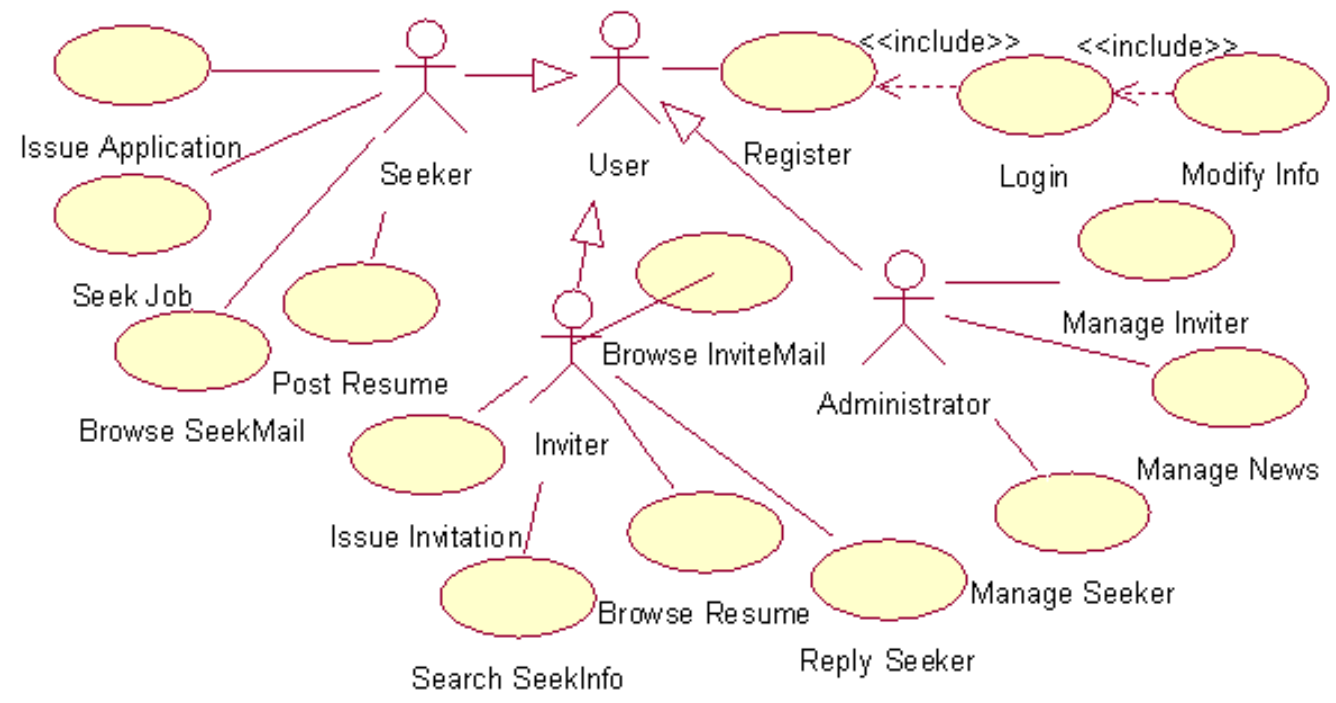

Figure 1. Use case diagram of the system

\section{Class Design}

1) Identify the classes in the system and their property and operation.

Here, only part of entity classes in the system is analyzed, including User, Seeker, Inviter, Administrator, Application, Invitation, Resume and News.

2) Identify the relationship between classes

Through analysis, the relationship between entity classes in the "employment and recruitment system" is shown in Table I. 
Table I. Relationship among the entity classes of the system

\begin{tabular}{c|c|c|c}
\hline No. & Class A & Class B & Relationship between class A and class B \\
\hline 1 & User & Seeker & Generalization \\
\hline 2 & User & Inviter & Generalization \\
\hline 3 & User & Administrator & Generalization \\
\hline 4 & Administrator & News & Association \\
\hline 5 & Seeker & Resume & Association \\
\hline 6 & Seeker & Invitation & Association \\
\hline 7 & Seeker & Application & Association \\
\hline 8 & Seeker & News & Association \\
\hline 9 & Inviter & Resume & Association \\
\hline 10 & Inviter & Invitation & Association \\
\hline 11 & Inviter & Application & Association \\
\hline 12 & Inviter & News & Association \\
\hline
\end{tabular}

The class diagram of the system can be generated based on the relationship between the classes as above. Since the general class diagram is too complicated, it is divided into three sub-diagrams as follows: Manage sub-diagram (shown in Fig. 2), Seek sub-diagram (shown in Fig. 3), and Invite sub-diagram (shown in Fig. 4).

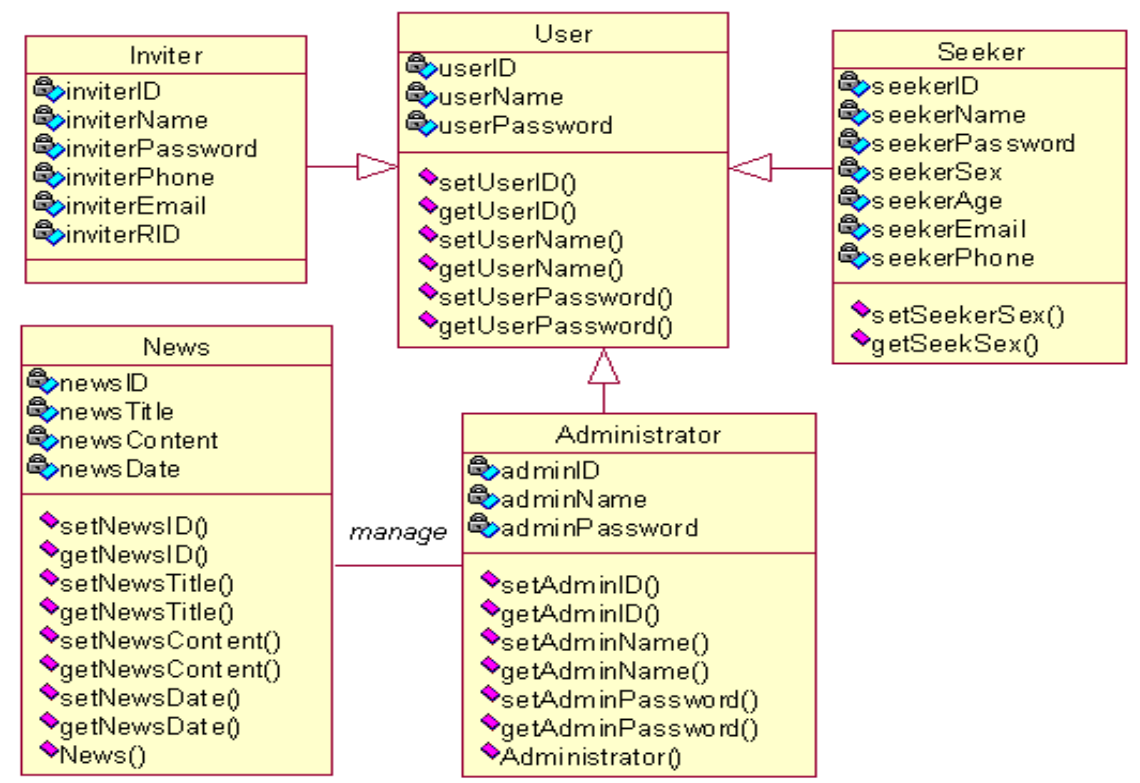

Figure 2. Manage sub-diagram

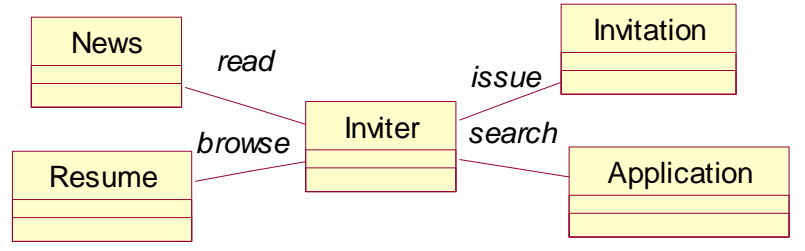

Figure 3. Seek sub-diagram

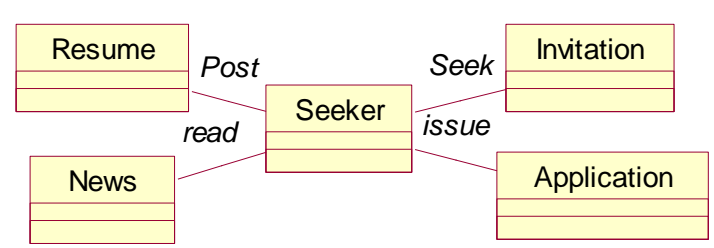

Figure 4. Invite sub-diagram

\section{Interactive Design}

1) Identify the objects, messages and other elements for specific scenes in the system and then draw a sequence diagram accordingly with Rational Rose.

Here, we analyze the scene of "Register" and build the model of it, and the sequence diagram 
is shown in Fig. 5.

2) Capture the activities of designated objects and use cases and then draw an activity diagram accordingly with Rational Rose.

Taking the activity of "update info" for example, the activity chart is shown in Fig. 6.

\section{Conclusion}

This paper analyzes and designs the employment and recruitment system with UML language. In the process of requirements analysis, we first capture the system participants, use cases and the relationship between them5, and then describe them with a use case diagram; in the process of static design, we identify the classes in the system and their relationship, which are presented in a class diagram; and in the process of dynamic design, taking "Register" and "Modify Info", two main use cases in the employment and recruitment system for example, we identify the objects, messages, activities and other elements in the system, which are illustrated in a sequence diagram and an activity diagram.

Our subsequent work will be writing object-oriented programs with development platform and languages on the basis of this model, which will not be detailed here.

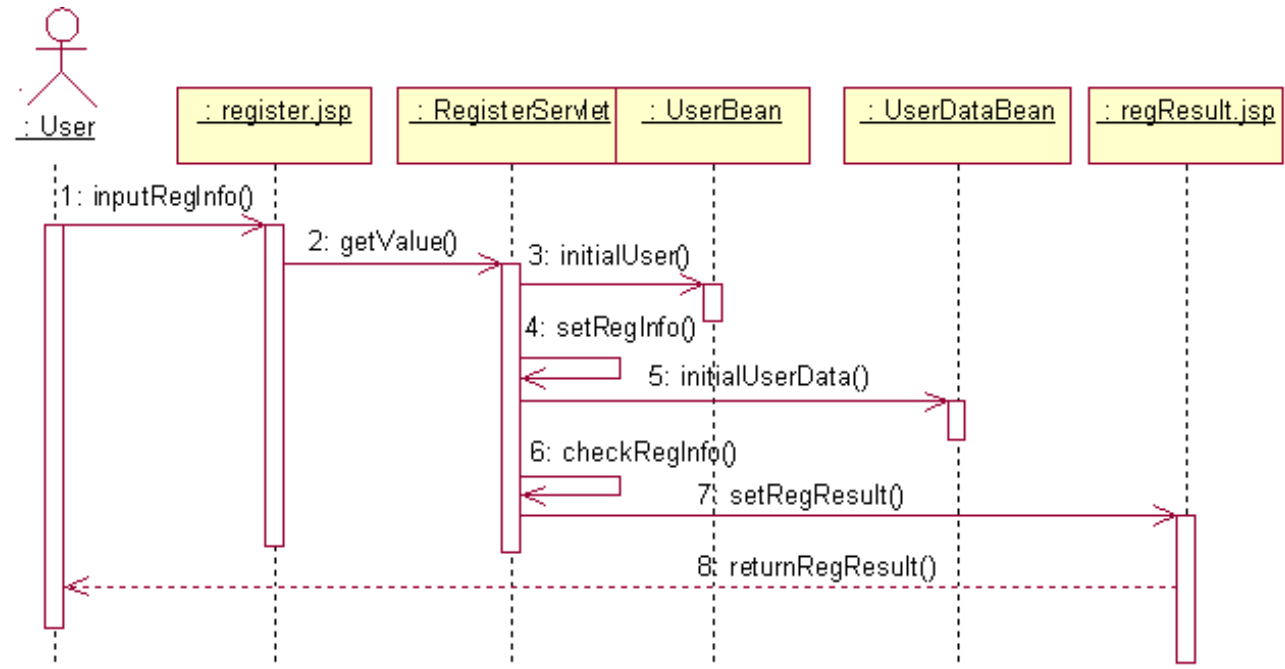

Figure5. sequence diagram of "Register"

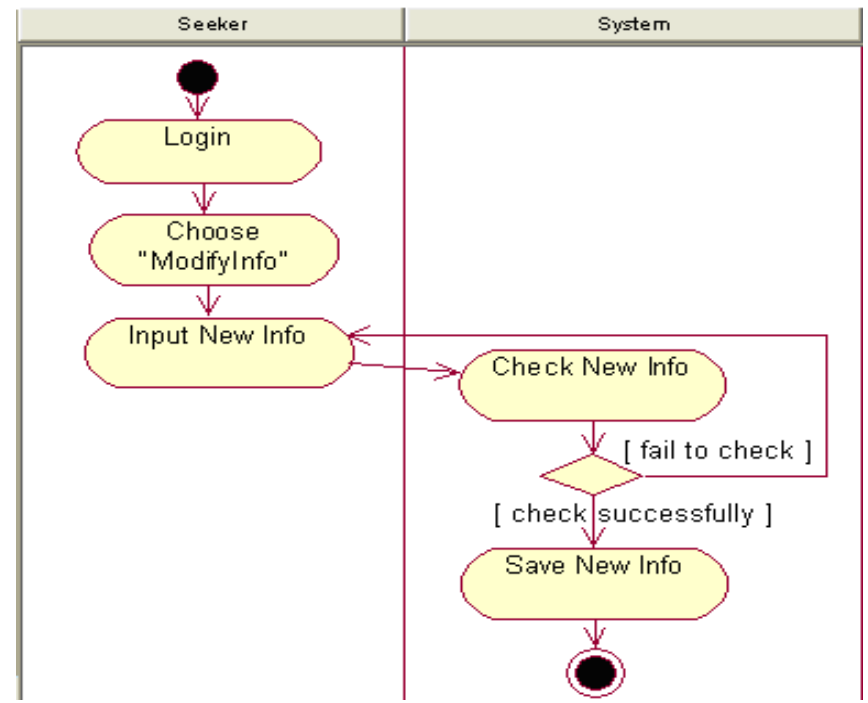

Figure 6. activity diagram of "Modify Info" 


\section{References}

[1]H.J.Tang, Training Tutorial of UML Foundation and Rose Modeling, Tsinghua University Press, Beijing, 2011.

[2]X.F.Wu, Design of Online Job Application \& Recruitment System Based on ASP.NET, Journal Of Nantong Vocational \& Technical Shipping College, 14(6):53-56.

[3]Ch.L.Zhao, Trainning Tutorial of Software Engineering, Publishing House of Electronics Industry, Beijing, 2007.

[4]Craig Larman. UML and Model Application, Machinery Industry Press, Beijing, 2009.

[5] J.L.Mo, S.X.Wang, Design and Implementation of IT Industry Recruitment Network Based on B/S, Journal of Changsha University, 27(3):57-59. 